\title{
Incidence and risk factors for failed medical management of spinal epidural abscess: a systematic review and meta-analysis
}

\author{
Alexandra Stratton, MD, ${ }^{1,2}$ Karla Gustafson, MD, ${ }^{1}$ Kenneth Thomas, MD, ${ }^{1,2}$ and \\ Matthew T. James, MD ${ }^{1,3}$ \\ Departments of ${ }^{1}$ Community Health Sciences, ${ }^{2}$ Surgery, and ${ }^{3}$ Medicine, Cumming School of Medicine, University of Calgary, \\ Alberta, Canada
}

\begin{abstract}
OBJECTIVE Spinal epidural abscess (SEA) is a life-threatening infection. It is uncertain whether medical versus surgical treatment is the ideal initial approach for neurologically intact patients with SEA. Recent evidence demonstrates that initial medical management is increasingly common; however, patients who ultimately require surgery after failed medical management may have a worse prognosis than those whose treatment was initially surgical. The primary objective of this study was to establish the current incidence of failed medical management for SEA. The secondary aim was to identify risk factors associated with the failure of medical management.
\end{abstract}

METHODS The authors conducted a systematic review and meta-analysis by searching electronic databases (MEDLINE, Embase, CINAHL, and PubMed), recent conference proceedings, and reference lists of relevant articles. Studies that reported original data on consecutive adult patients with SEA treated medically were eligible for inclusion.

RESULTS Twelve studies met the inclusion criteria, which included a total of 489 medically treated patients with SEA. Agreement on articles for study inclusion was very high between the reviewers (kappa 0.86). In a meta-analysis, the overall pooled risk of failed medical management was $29.3 \%(95 \% \mathrm{Cl} 21.4 \%-37.2 \%)$ and when medical to surgical crossover was used to define failure the rate was $26.3 \%(95 \% \mathrm{Cl} 13.0 \%-39.7 \%)$. Only 6 studies provided data for analysis by intended treatment, with a pooled estimate of $35.1 \%(95 \% \mathrm{Cl} 15.7 \%-54.4 \%)$ of failed medical management. Two studies reported predictors of the failure of medical management.

CONCLUSIONS Although the incidence of failed medical management of SEA was relatively common in published reports, estimates were highly heterogeneous between studies, thus introducing uncertainty about the frequency of this risk. A consensus definition of failure is required to facilitate comparison of failure rates across studies.

http://thejns.org/doi/abs/10.3171/2016.6.SPINE151249

KEY WORDS spinal epidural abscess; medical management; failure; antibiotics; spine surgery; infection

$\mathrm{S}$ PINAL epidural abscess (SEA) is a life-threatening infection involving the epidural space. Current mortality rates are estimated at 5\%-16\% worldwide, ${ }^{8,16}$ and fewer than half of patients who survive this infection fully recover. ${ }^{16}$ While SEA is a relatively rare diagnosis, its incidence has nearly doubled in the last 50 years possibly because of increased intravenous drug abuse, long-term vascular access in conjunction with spinal instrumentation, the aging population, and the availability of MRI, which has increased the sensitivity of detection. . $^{8,13,17,18}$

Once a diagnosis of SEA has been made, treatment is largely based on the presence or absence of acute or progressive neurological deficits. ${ }^{3}$ In the former instance, there is little debate that urgent surgical debridement is required. ${ }^{3}$ There is disagreement, however, about the optimum initial therapeutic approach for patients without neurological deficit. ${ }^{22}$ A recent systematic review revealed that since 1999 there has been an overall trend toward more frequent medical management for neurologically intact patients with SEA, although practices remain highly variable. ${ }^{3}$ Nonsurgical management consists of several weeks of intravenous antibiotics and close clinical follow-up. Pa-

ABBREVIATIONS MRSA = methicillin-resistant Staphylococcus aureus; $\mathrm{SEA}=$ spinal epidural abscess; $\mathrm{TB}=$ tuberculosis .

SUBMITTED October 19, 2015. ACCEPTED June 6, 2016.

INCLUDE WHEN CITING Published online September 16, 2016; DOI: 10.3171/2016.6.SPINE151249. 
tients who ultimately require surgery after failed medical management may have a worse prognosis than those whose treatment was initially surgical. ${ }^{9}$ Data on the risk of failed medical management for SEA is important to inform clinical decision making and enable physicians to communicate to patients their risk of treatment failure when discussing options.

We performed a systematic review and meta-analysis to establish the current incidence of failed medical management for SEA. The secondary aim of our systematic review was to identify risk factors associated with the failure of medical management for SEA to aid clinicians in predicting who can safely be treated medically.

\section{Methods}

We followed a prespecified protocol for study selection and analysis of results as well as the reporting recommendations outlined in the Meta-analysis of Observational Studies in Epidemiology (MOOSE) guidelines. ${ }^{20}$

\section{Data Sources and Searches}

We searched the electronic databases MEDLINE, Embase, CINAHL, and PubMed from inception to October 27, 2014. Our search in MEDLINE included medical subject headings (MeSH) for the study population theme "central nervous system infections" or "bacterial central nervous system infections" or "epidural abscess." Our search terms for the exposure theme included the exploded MeSH "operative surgical procedure" or "anti-bacterial agents" and title and abstract key word terms "treatment" or "management." We combined the results of the study population search theme with that of the exposure search theme using the Boolean operator "AND." Using Embase subject headings (Emtree) and title and abstract key word terms, we conducted an identical search of Embase restricted to adults. We also searched CINAHL for the term "epidural abscess" as well as PubMed by using "CNS infections" and "antibacterial agents" or "operative surgical procedure" for 2014 to identify new articles not yet indexed in MEDLINE. All citations identified through database searching were imported into EndNote X7 citation software and duplicates were removed.

In addition, we searched the reference lists of relevant articles, and the 2012-2014 conference proceedings from the North American Spine Society and International Society for the Study of the Lumbar Spine. Non-English articles included for full-text review were translated as required. All citations were screened, and full-text reviews were conducted by 2 independent physician reviewers. We contacted authors for additional information when necessary and for data from identified abstracts not published as full manuscripts.

\section{Study Selection}

Articles were selected for inclusion using a 2-step process. In the first step, we screened abstracts for inclusion if a study reported original data on the consecutive selection of adult patients with SEA and included patients treated medically. In the second step, we conducted full-text reviews of articles. Our primary outcome of failed medi- cal management was considered broadly, acknowledging the variability in clinical definitions of this outcome. We included within the definition of failed medical management those studies reporting outcomes of persistent severe neurological deficits, poor clinical outcome, worse clinical outcome, death, or the need for surgery in patients who had received antibiotic therapy. Articles were excluded after full-text review if no specific information on the outcome of medically treated patients was provided, 10 or fewer medically treated patients were described, nonconsecutive patients were included, or specific etiologies such as postoperative infections or no original data were provided.

\section{Data Extraction}

During full-text review, we extracted data on the criteria for medical management and the number of patients treated medically and surgically. Patients treated only with percutaneous abscess drainage were considered to have received medical management. We collected data from studies for secondary analysis of the primary outcome in which the failure of medical management was based solely on the need for a medically managed patient to cross over to surgical treatment because of a persistent or deteriorating neurological or medical condition. Additional information was collected on demographics, patient comorbidities and immune status, diagnostic procedures, and outcome information where available. When provided, all data on predictors of failed medical management were extracted for analysis of the secondary outcome.

\section{Quality Assessment}

We evaluated the quality of each included study based on a 6-item checklist adapted from Altman's framework for assessing the internal validity of prognostic studies. ${ }^{2}$ This checklist comprised the following parameters: patient inclusion criteria defined, diagnostic criteria described, patient characteristics described, treatment described, outcome known, and follow-up described. Two reviewers independently graded the 6 items as "full" (fully described) or "poor" (poorly described). We considered a study to fully describe patient inclusion criteria if all patients with SEA over a given time period were included or if the inclusion and exclusion criteria were specified. We considered a full description of diagnostic criteria as one explicitly stating how patients were diagnosed (for example, clinical, lab, culture/biopsy, or imaging results). Full reporting of patient characteristics meant that demographic information as well as patient comorbidities were provided. Treatment was rated as fully described for studies that outlined the intended treatment course, crossover if any, duration of time to operation, type of operation, duration of antibiotic treatment, number of patients undergoing percutaneous drainage, and how patients were classified in terms of treatment group in the analysis. Outcome was fully described if outcome by treatment group, including the number of deaths in each group, was presented. Studies that reported the duration of follow-up as not less than 6 weeks and provided an explanation of outcome assessment were rated "full" for follow-up description. 


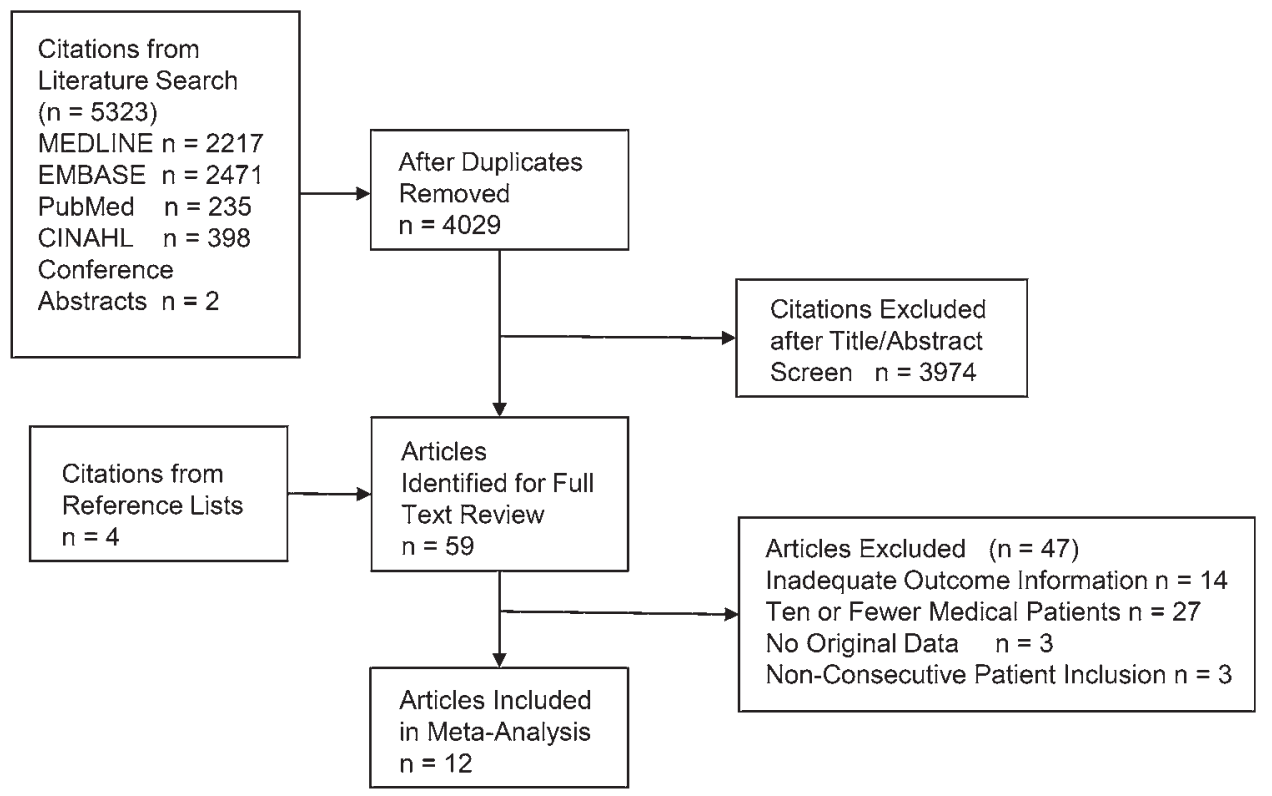

FIG. 1. Flow chart of study selection.

\section{Data Synthesis and Analysis}

We determined the pooled proportion of patients with failed medical management by using a random effects model. This model was chosen given the variability in the preferred treatment of SEA at different centers, the spectrum of symptoms on presentation, and the inconsistent treatment regimens. Heterogeneity was quantified using the $\mathrm{I}^{2}$ statistic. For studies with overlapping patients, only the larger study was included in a pooled analysis. Stratified and sensitivity analyses were performed to investigate potential causes of heterogeneity. Stratified analysis was conducted based on the inclusion of patients with tuberculosis (TB), the included percentage of patients with methicillin-resistant Staphylococcus aureus (MRSA) and diabetes, and the quality of the study treatment description. Sensitivity analyses were performed to replace the larger of the duplicate cohorts with the smaller cohort and to exclude studies that included pediatric patients. All analyses were performed using Stata version 13 software with alpha set at 0.05 .

\section{Results}

\section{Identification of Studies}

Our electronic bibliographic database search identified 4029 unique citations (Fig. 1). Screening of title and abstracts by both reviewers yielded 59 articles for full-text review. After the full-text review, 12 observational studies were included for analysis. Agreement between reviewers was very high (Cohen's kappa coefficient of 0.86 for interrater agreement). ${ }^{15}$

\section{Study Characteristics}

All included studies reported the number of patients considered to have failure of medical management, with this outcome variably defined across studies (Table 1). Two studies had an apparent overlap of patients older than 50 years of age between 1999 and 2007.110 All studies reported the proportion of patients with positive cultures, either from blood or abscess aspirate, as well as the most common pathogens. The proportion of patients with a positive culture ranged from $69 \%$ to $100 \%$, with a mean of $86 \%$ across the studies. However, only one study detailed the pathogen by treatment group. Six studies specifically reported those patients in the medically treated group who had crossed over to the surgery group as a result of worsening symptoms. ${ }^{7,11,12,14,18,19}$ In 5 of these studies, it was possible to determine the proportion in whom treatment had failed based on a composite of requisite surgery after medical management, worsening clinical status, or death in order to enhance consistency across studies for comparison. ${ }^{7,11,12,18,19}$

\section{Study Quality}

The reporting of inclusion criteria and patient characteristics was well done across studies (Table 2). Only 1 study excluded patients presenting with neurological compromise of more than 48 hours, which is most applicable to the clinical practice of offering medical therapy to patients without early indications for surgery. ${ }^{12}$ The description of treatment was poor in most studies, with only 4 noting the intended treatment plan., ${ }^{7,14,18}$ Eight studies reported results based on the final treatment received without specifying whether the final treatment was the intended one.,14$6,10,11,19,21$ Of these 8 studies, 3 specified the time from diagnosis to surgery, which ranged from 0 to 42 days. ${ }^{4-6}$ Only 7 studies reported the number of patients who had died in the 2 treatment groups. ${ }^{1,5,6,10,12,18,19}$ The description of follow-up was poor, with 10 studies failing to report a minimum patient follow-up of 6 weeks or more to ensure resolution of infection. ${ }^{1,4-7,10,14,18,19,21}$ 


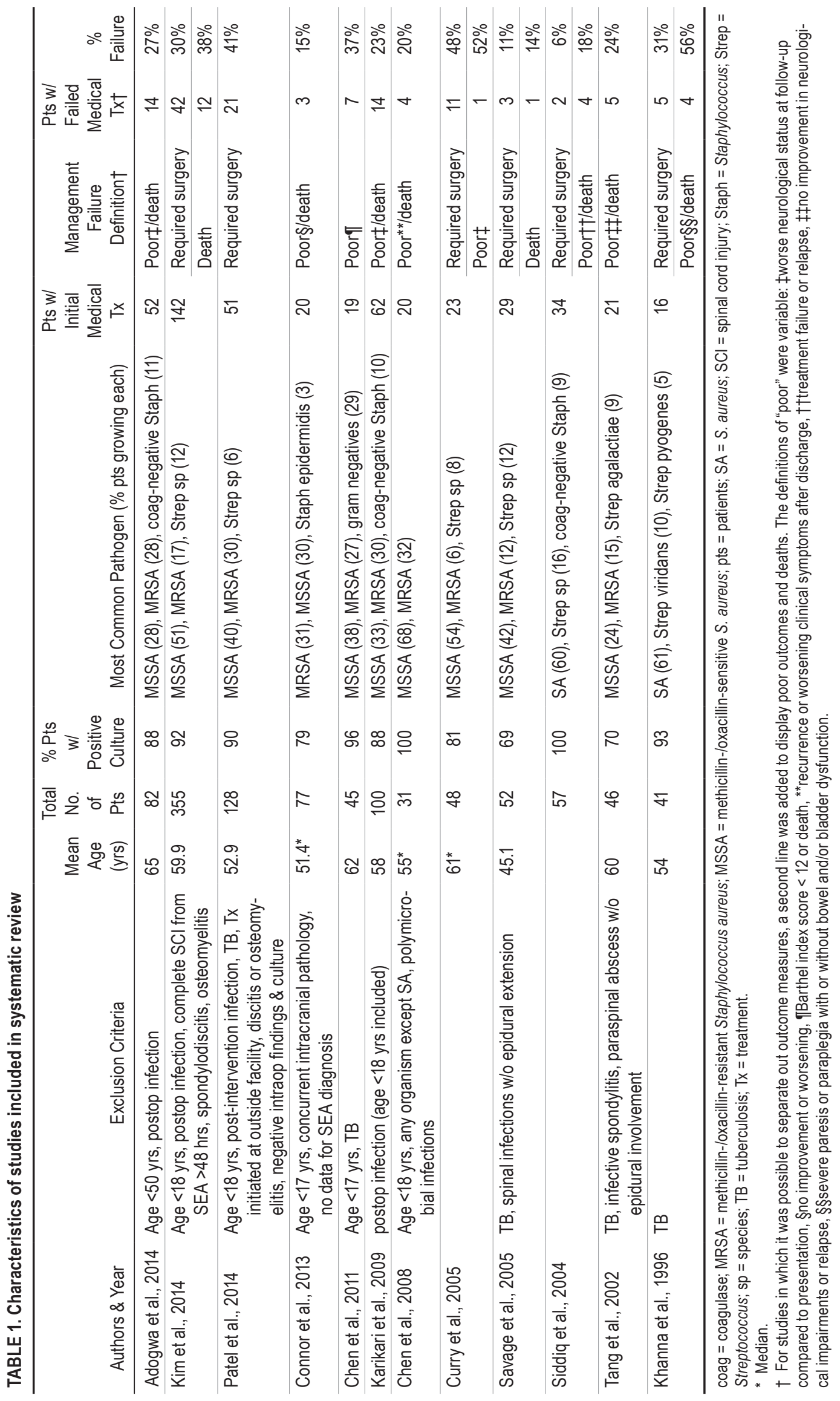


TABLE 2. Study quality assessment

\begin{tabular}{lcccccc}
\hline \multicolumn{1}{c}{ Authors \& Year } & Inclusion Criteria & Diagnostic Criteria & Patient Characteristics & Tx & Outcome & FU \\
\hline Adogwa et al., 2014 & Full & Full & Full & Poor & Full & Poor \\
\hline Chen et al., 2011 & Full & Full & Full & Poor & Partial & Poor \\
\hline Chen et al., 2008 & Full & Full & Full & Poor & Full & Poor \\
\hline Connor et al., 2013 & Full & Poor & Full & Poor & Full & Poor \\
\hline Curry et al., 2005 & Full & Poor & Full & Full & Partial & Poor \\
\hline Karikari et al., 2009 & Full & Full & Full & Poor & Full & Poor \\
\hline Khanna et al., 1996 & Full & Poor & Full & Poor & Poor & Poor \\
\hline Kim et al., 2014 & Full & Full & Full & Full & Full & Poor \\
\hline Patel et al., 2014 & Full & Poor & Full & Full & Poor & Poor \\
\hline Savage et al., 2005 & Full & Partial & Full & Full & Full & Poor \\
\hline Siddiq et al., 2004 & Full & Poor & Full & Poor & Full & Poor \\
\hline Tang et al., 2002 & Full & Partial & Full & Poor & Poor & Poor \\
\hline
\end{tabular}

$\mathrm{FU}=$ follow-up

\section{Pooled Proportion of Failure of Medical Therapy}

We identified 11 studies that reported data on the proportion of patients in whom medical management had failed, with failure defined as a poor outcome, a worse neurological status, and death, in addition to the need for surgery. The pooled proportion of failed medical management was 29.3\% (95\% CI 21.4\%-37.2\%; Fig. 2; Adogwa et al. study excluded from analysis since its patients were also reported in Karikari et al.). The $\mathrm{I}^{2}$ value of $69.5 \%$ indicated moderate heterogeneity. We performed a secondary analysis including 6 studies that reported medical to surgical crossover as a definition of failure, $7,11,12,14,18,19$ the pooled proportion of failed medical management by this definition was $26.3 \%$ (95\% CI $13.0 \%-39.7 \%$ ), with an $\mathrm{I}^{2}$ value of $87 \%$ indicating high heterogeneity (Fig. 3).

\section{Sensitivity Analysis}

We conducted sensitivity analyses to test on the pooled estimate the effect of removing the single study that included pediatric patients, ${ }^{10}$ the study that was restricted to patients over the age of 50 years, ${ }^{1}$ and the study that included only $S$. aureus infections..$^{5}$ Removing each of these studies individually did not significantly change the pooled risk estimate of $29.3 \%$ (95\% CI $21.4 \%-37.2 \%$ ). When we excluded the larger study by Karikari et al., ${ }^{10}$ which included some of the same patients as those in the

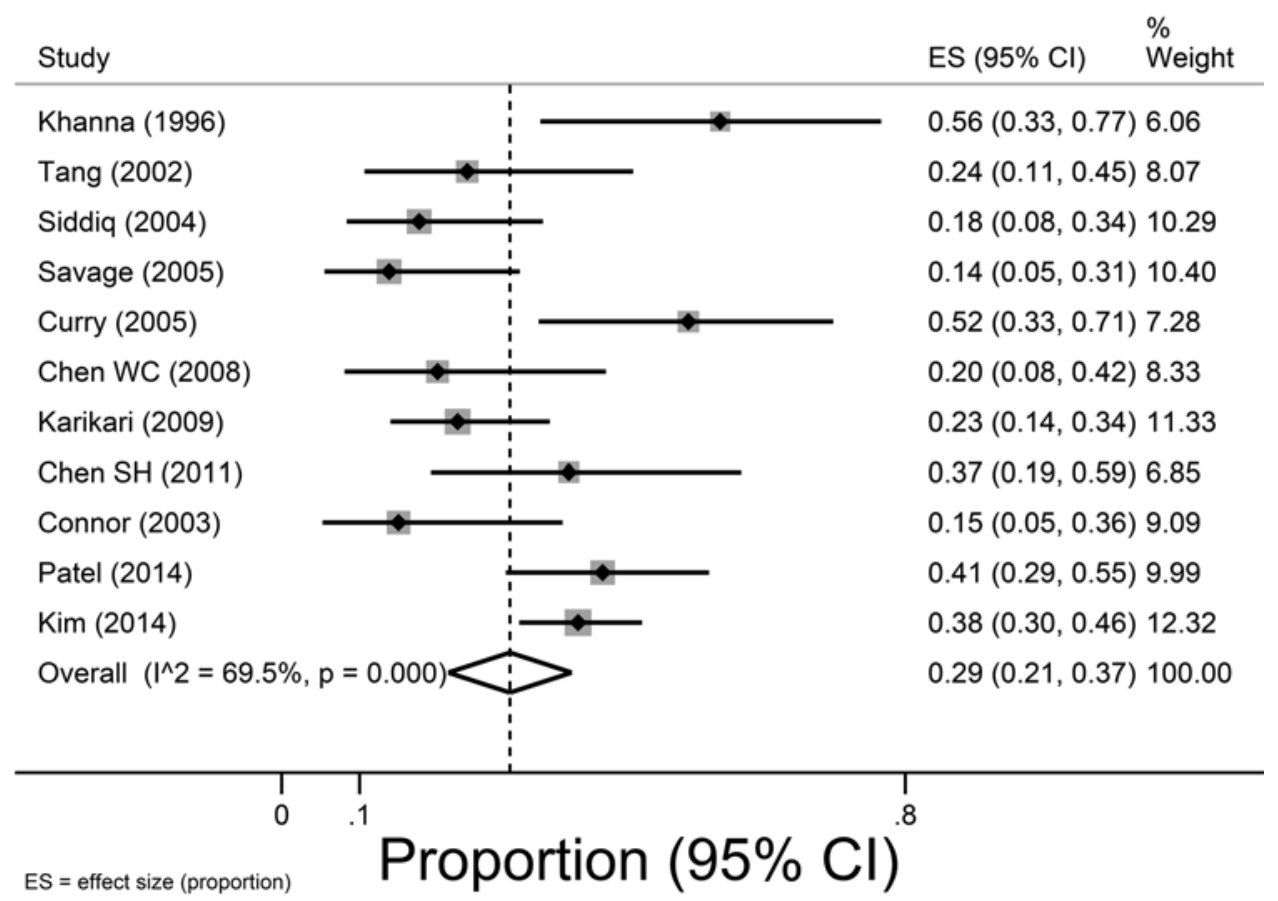

FIG. 2. Forest plot of the proportion of patients with failed medical management based on any definition of failure reported by a study. 


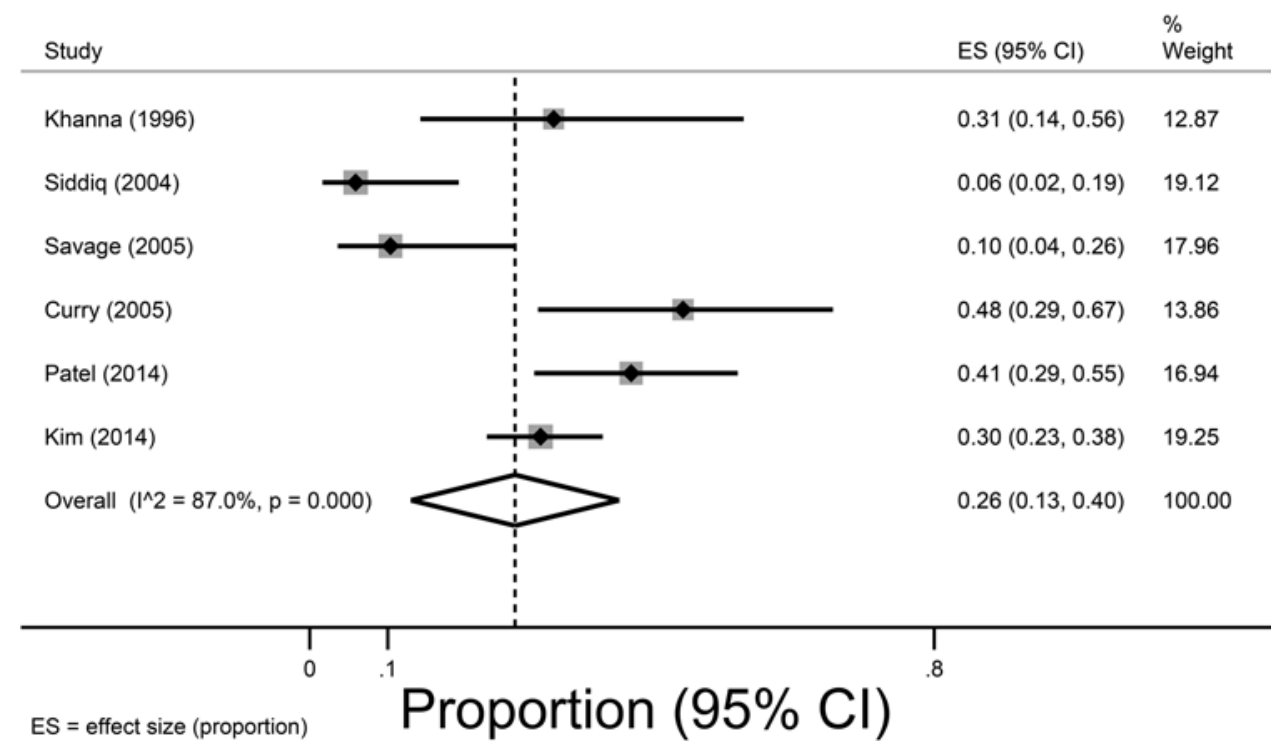

FIG. 3. Forest plot of proportion of patients with failed medical management based on failure defined as requiring surgery.

Adogwa et al. study, and replaced it with the Adogwa study, ${ }^{1}$ the pooled result remained similar $(29.8 \%, 95 \%$ CI 21.9-38.7\%).

\section{Stratified Analysis}

To investigate potential sources of heterogeneity, we performed stratified analyses and meta-regression. When studies were stratified by those with more versus less than $20 \%$ MRSA infection rates (no studies had exactly $20 \%$ MRSA; among all treatment groups), we found no difference in the risk of failed medical management between the 2 strata (Table 3), with the proportion of MRSA (high or low) accounting for only $3.4 \%$ of the heterogeneity observed. Stratification of studies by the inclusion or exclusion of patients with TB infection showed no difference in the risk for medical treatment failure and resulted in an increase in heterogeneity between studies within strata.
With stratification according to the sufficiency of treatment description, the difference in the estimated failure of medical management between those studies with full versus poor treatment descriptions was not statistically significant, with $10.0 \%$ of the heterogeneity accounted for by this variable. When studies were stratified as those with more versus less than $20 \%$ of patients with diabetes (among all treatment groups), we did not find a significant difference in the risk of failed medical management between the 2 strata. The proportion of patients with diabetes accounted for only $2.9 \%$ of the heterogeneity observed.

\section{Publication Bias}

A funnel plot and Begg's test $(\mathrm{p}=0.27)$ provided no evidence of statistically significant small study effects; however, we noted more studies with fewer patients reporting higher proportions of failed medical management (Fig. 4).

TABLE 3. Stratified analyses

\begin{tabular}{|c|c|c|c|c|c|}
\hline Variable & $\begin{array}{l}\text { No. of } \\
\text { Studies }\end{array}$ & $\begin{array}{c}\text { Total No. of } \\
\text { Patients in Study }\end{array}$ & $\begin{array}{c}\% \text { w/ FMT } \\
(95 \% \mathrm{Cl})\end{array}$ & $\begin{array}{c}\text { Meta-Regression } \\
\text { p Value }\end{array}$ & $\begin{array}{c}\% \\
\text { Heterogeneity }\end{array}$ \\
\hline Treatment description & & & & 0.29 & 10.0 \\
\hline Full & 4 & 583 & $35.1(15.7-54.4)$ & & \\
\hline Poor & 7 & 397 & $25.4(13.1-37.6)$ & & \\
\hline MRSA & & & & 0.32 & 3.4 \\
\hline$>20 \%$ & 4 & 235 & $24.1(4.0-44.1)$ & & \\
\hline$<20 \%$ & 5 & 629 & $33.1(20.2-46.1)$ & & \\
\hline TB patients & & & & 0.82 & -19.0 \\
\hline Included & 5 & 637 & $28.3(7.9-48.8)$ & & \\
\hline Excluded & 6 & 343 & $30.5(16.1-44.9)$ & & \\
\hline Diabetes & & & & 0.38 & 2.9 \\
\hline$>20 \%$ & 7 & 792 & $32.3(12.1-52.5)$ & & \\
\hline$<20 \%$ & 4 & 188 & $24.0(7.9-40.2)$ & & \\
\hline
\end{tabular}

FMT = failed medical treatment. 


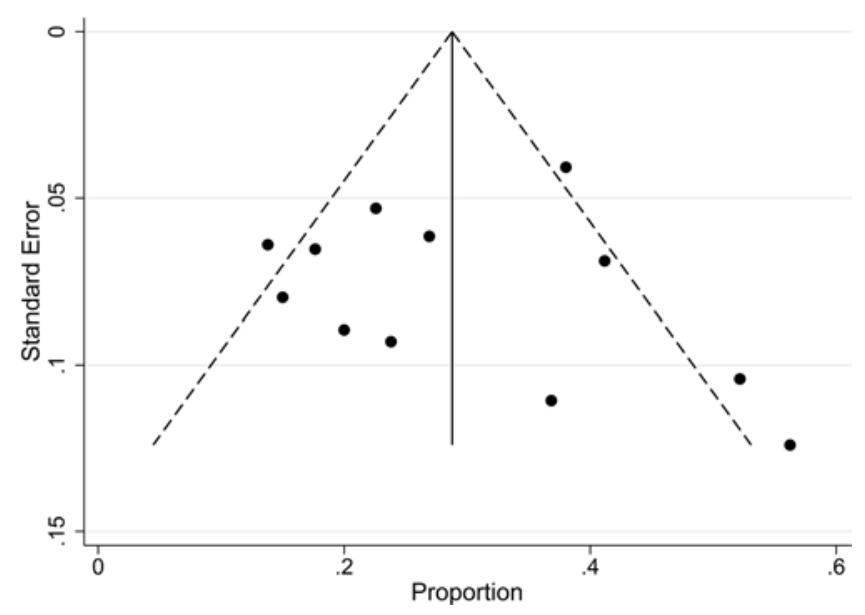

FIG. 4. Funnel plot with pseudo-95\% confidence limits.

\section{Predictors of Medical Treatment Failure}

Two studies reported predictors of failed medical management (Table 4). Kim et al. reported that an age greater than 65, diabetes, MRSA, and neurologic impairment were significant risk factors for medical treatment failure in multivariable models, ${ }^{12}$ whereas Patel et al. reported positive blood cultures, diabetes, WBC $>12.5$, and c-reactive protein $>115$ as significant univariable predictors. ${ }^{14}$ The odds ratios associated with each criterion are reported in Table 4.

\section{Discussion}

Our systematic review of the incidence of failed medical management for SEA identified 12 observational studies with at least 10 patients initially treated medically. In a pooled analysis of 11 studies reporting independent patients, we found that the proportion of SEA patients with failed medical management was $29 \%$ with moderate heterogeneity between studies. When we defined treatment failure based on 6 studies that reported on patients requiring surgery after initial attempts at medical management, the resulting estimate of the proportion of failure was $26 \%$ with high heterogeneity between studies. In stratified analyses to explore potential sources of heterogeneity, we found that studies reporting outcomes according to intended treatment strategy documented higher risks of treatment failure than those reporting outcomes based on the final treatment received (35\% vs 25\%). Our findings highlight the limitation of studies that do not report outcomes based on intended treatment strategy, which probably underestimate the failure rate of initial medical management.

We found significant heterogeneity between studies that could not be fully explained in stratified or sensitivity analyses. Further sources of clinical heterogeneity between studies that we were unable to test for include the delay between onset of symptoms and diagnosis of SEA, anatomical location of abscess(es), timing of the first dose of antibiotics (before or after cultures), use of appropriate pathogen-specific antibiotics, type and virulence of an organism (other than MRSA and TB), patient immune
TABLE 4. Published predictors for failed medical management, with associated ORs

\begin{tabular}{cc}
\hline \multicolumn{1}{c}{ Study \& Predictor } & OR \\
\hline Kim et al., 2014 & Multivariable \\
\hline Age $>65$ yrs & 2.5 \\
\hline Diabetes & 2.5 \\
\hline MRSA & 3.8 \\
\hline Neurological impairment (incomplete or & 15.2 \\
complete cord injury) & \\
\hline Patel et al., 2014 & Univariable \\
\hline Positive blood culture & 3.5 \\
\hline Diabetes mellitus & 3.8 \\
\hline Leukocytosis $>12.5$ & 3.3 \\
\hline CRP $>115$ & 4.7 \\
\hline
\end{tabular}

$\mathrm{CRP}=\mathrm{C}$-reactive protein.

status, use of percutaneous drainage and/or aspiration, criteria for selecting treatment strategy, rationale for crossing over to surgery, time to surgery in the surgical group, outcome definitions, and duration of follow-up. Two reviews on the treatment of SEA have been recently published; ${ }^{3,22}$ however, neither was specifically designed to study the failure of medical management in this disease. Arko et al. reported large variability in the proportion of medical treatment failure (defined as requiring surgery) ranging from $6 \%$ to $49 \%$ across studies. ${ }^{3}$ This range is consistent with the estimate we identified using the same definition of treatment failure $(26 \%)$. Tuchman et al. did not report the proportion of failure..$^{22}$

The strengths of our systematic review and meta-analysis include a comprehensive literature search of both English and non-English articles and high interrater agreement for study selection. However, our systematic review is limited by the quality of the published literature. Studies on the management of SEA have all been retrospective in nature as the incidence of SEA is small. The definition of treatment failure has varied considerably across studies, suggesting a need for consensus on the definition of this outcome to facilitate outcomes research. Furthermore, it is critical for authors to specify whether patients are treated medically because of a lack of surgical indication(s) or because of comorbidities precluding surgical intervention. Another important limitation is the small proportion of studies reporting outcome by intended treatment course. There was a lengthy delay from the time of diagnosis to surgery (up to 42 days) in the 3 studies that reported this information. ${ }^{4-6}$ This delay suggests that the intended treatment course in an unspecified number of patients was probably not surgical, despite the fact that these patients were included in the surgical group for outcome reporting.

This is the first meta-analysis to report the proportion of failed medical management of SEA by intended treatment course and by failure definition. Our secondary objective was to identify risk factors associated with the failure of medical management for SEA to aid clinicians in predicting which patients can be safely treated medically. We found that in 2014 the 2 largest studies on SEA both addressed a gap in the literature regarding the variables that 
predict failed medical management of SEA. These studies were the first to publish criteria for predicting medical treatment failure based on regression analyses of multiple possible contributory patient characteristics. However, the studied predictor variables differed, resulting in the identification of distinct prediction criteria (Table 3) that do not overlap aside from the presence of diabetes. Further research is needed to synthesize this information to derive and validate more robust multivariable prediction models.

The results of this meta-analysis are generalizable to all patients with medically treated SEA; however, a more clinically useful estimate would be based on a population excluding patients who are not surgical candidates because of medical comorbidities and patients with prolonged neurological deficit (greater than 36-48 hours). Inclusion of these patients in studies comparing medical and surgical management is problematic because such patients have a poor prognosis regardless of treatment. By excluding patients with symptoms of complete spinal cord injury present for more than 48 hours on presentation, the study by $\mathrm{Kim}$ et al. is the most relevant to initial treatment decisions for early surgical versus medical management among patients eligible for either approach. ${ }^{12}$ That study reported the proportion of failed medical management as 30\% (requiring surgery) and 38\% (including deaths). These rates of failed medical management are extremely high and reinforce the need for further research to help physicians identify patients who can be appropriately treated medically. Future research should focus on the subset of SEA patients without prolonged neurological deficit and without contraindications to surgery and employ standardized outcome reporting by using validated functional outcome questionnaires. These standards would make results comparable across studies and assist in clinical decision making. Further research to validate the prognostic accuracy of the prediction criteria suggested by Kim et al. and Patel et al. is warranted.

\section{Conclusions}

In summary, we found high variability across studies with respect to rationale for the chosen treatment, definition of treatment failure, and outcome reporting for SEA. Although we found that the incidence of failed medical management of SEA was relatively common in published reports, estimates were highly heterogeneous between studies, thus introducing uncertainty about the frequency of this risk. Future research in this area should clearly document the intended treatment course for all patients, the time delay between initiation of antibiotic treatment and surgery when required, and outcomes based on intended treatment. Furthermore, it is critical to develop a consensus definition of failure to facilitate comparisons of failure rates across studies.

\section{References}

1. Adogwa O, Karikari IO, Carr KR, Krucoff M, Ajay D, Fatemi P, et al: Spontaneous spinal epidural abscess in patients 50 years of age and older: a 15-year institutional perspective and review of the literature: clinical article. J Neurosurg Spine 20:344-349, 2014

2. Altman DG: Systematic reviews of evaluations of prognostic variables. BMJ 323:224-228, 2001

3. Arko L IV, Quach E, Nguyen V, Chang D, Sukul V, Kim BS: Medical and surgical management of spinal epidural abscess: a systematic review. Neurosurg Focus 37(2):E4, 2014

4. Chen SH, Chang WN, Lu CH, Chuang YC, Lui CC, Chen SF, et al: The clinical characteristics, therapeutic outcome, and prognostic factors of non-tuberculous bacterial spinal epidural abscess in adults: a hospital-based study. Acta Neurol Taiwan 20:107-113, 2011

5. Chen WC, Wang JL, Wang JT, Chen YC, Chang SC: Spinal epidural abscess due to Staphylococcus aureus: clinical manifestations and outcomes. J Microbiol Immunol Infect 41:215-221, 2008

6. Connor DE Jr, Chittiboina P, Caldito G, Nanda A: Comparison of operative and nonoperative management of spinal epidural abscess: a retrospective review of clinical and laboratory predictors of neurological outcome. J Neurosurg Spine 19:119-127, 2013

7. Curry WT Jr, Hoh BL, Amin-Hanjani S, Eskandar EN: Spinal epidural abscess: clinical presentation, management, and outcome. Surg Neurol 63:364-371,2005

8. Darouiche RO: Spinal epidural abscess. N Engl J Med 355:2012-2020, 2006

9. Hlavin ML, Kaminski HJ, Ross JS, Ganz E: Spinal epidural abscess: a ten-year perspective. Neurosurgery 27:177-184, 1990

10. Karikari IO, Powers CJ, Reynolds RM, Mehta AI, Isaacs RE: Management of a spontaneous spinal epidural abscess: a single-center 10-year experience. Neurosurgery 65:919-924, 2009

11. Khanna RK, Malik GM, Rock JP, Rosenblum ML: Spinal epidural abscess: evaluation of factors influencing outcome. Neurosurgery 39:958-964, 1996

12. Kim SD, Melikian R, Ju KL, Zurakowski D, Wood KB, Bono $\mathrm{CM}$, et al: Independent predictors of failure of nonoperative management of spinal epidural abscesses. Spine J 14:16731679,2014

13. Nussbaum ES, Rigamonti D, Standiford H, Numaguchi Y, Wolf AL, Robinson WL: Spinal epidural abscess: a report of 40 cases and review. Surg Neurol 38:225-231, 1992

14. Patel AR, Alton TB, Bransford RJ, Lee MJ, Bellabarba CB, Chapman JR: Spinal epidural abscesses: risk factors, medical versus surgical management, a retrospective review of 128 cases. Spine J 14:326-330, 2014

15. Posner KL, Sampson PD, Caplan RA, Ward RJ, Cheney FW: Measuring interrater reliability among multiple raters: an example of methods for nominal data. Stat Med 9:1103-1115, 1990

16. Reihsaus E, Waldbaur H, Seeling W: Spinal epidural abscess: a meta-analysis of 915 patients. Neurosurg Rev 23:175-205, 2000

17. Rigamonti D, Liem L, Sampath P, Knoller N, Namaguchi Y, Schreibman DL, et al: Spinal epidural abscess: contemporary trends in etiology, evaluation, and management. Surg Neurol 52:189-197, 1999

18. Savage K, Holtom PD, Zalavras CG: Spinal epidural abscess: early clinical outcome in patients treated medically. Clin Orthop Relat Res 439:56-60, 2005

19. Siddiq F, Chowfin A, Tight R, Sahmoun AE, Smego RA Jr: Medical vs surgical management of spinal epidural abscess. Arch Intern Med 164:2409-2412, 2004

20. Stroup DF, Berlin JA, Morton SC, Olkin I, Williamson GD, Rennie D, et al: Meta-analysis of observational studies in epidemiology: a proposal for reporting. Meta-analysis Of Observational Studies in Epidemiology (MOOSE) group. JAMA 283:2008-2012, 2000 
21. Tang HJ, Lin HJ, Liu YC, Li CM: Spinal epidural abscessexperience with 46 patients and evaluation of prognostic factors. J Infect 45:76-81, 2002

22. Tuchman A, Pham M, Hsieh PC: The indications and timing for operative management of spinal epidural abscess: literature review and treatment algorithm. Neurosurg Focus 37(2):E8, 2014

\section{Disclosures}

The authors report no conflict of interest concerning the materials or methods in this study or the findings specified in this paper.

\section{Author Contributions}

Conception and design: all authors. Acquisition of data: Stratton, Gustafson. Analysis and interpretation of data: James, Stratton, Gustafson. Drafting the article: Stratton, Gustafson. Critically revising the article: James, Thomas. Reviewed submitted version of manuscript: James, Thomas. Approved the final version of the manuscript on behalf of all authors: James. Study supervision: James, Thomas.

\section{Supplemental Information \\ Online-Only Content}

Supplemental material is available with the online version of the article.

Appendix Tables 1 and 2. http://thejns.org/doi/suppl/10.3171/ 2016.6.SPINE151249.

\section{Correspondence}

Matthew T. James, Division of Nephrology, Departments of Medicine and Community Health Sciences, University of Calgary, Foothills Medical Centre, 1403 29th St. NW, Calgary, AB T2N 2T9, Canada.email: mjames@ucalgary.ca. 